\title{
A unified approach for radiative losses and backscattering in optical waveguides
}

\author{
D. Melati, F. Morichetti and A. Melloni
}

Dipartimento di Elettronica, Informazione e Bioingegneria, Politecnico di Milano, via Ponzio 34/5, I-20133 Milano, Italy

E-mail: daniele.melati@polimi.it

Received 9 January 2014, revised 12 March 2014

Accepted for publication 12 March 2014

Published 9 April 2014

\section{Introduction}

Sidewall roughness is an unavoidable surface imperfection generated by the fabrication processes of optical waveguides which causes a local variation of the waveguide width and consequently a random spatial fluctuation of the refractive index profile. The interaction b etween the 1 ight in the waveguide and the sidewall roughness induces a coupling mechanism which transfers part of the optical power from the actual propagating mode(s) to all the other guided modes (propagating and counter-propagating) and radiative modes [1]. This phenomenon has been widely investigated in the literature and models have been proposed to estimate the extrinsic losses generated by the radiated power and by the backscattering along the waveguide [1-6]. Even if these models can produce very accurate results, they are generally not easy to apply to waveguides with an arbitrary cross-section and rarely reveal the profound nature of the interaction between the electromagnetic field and the roughness on the waveguide surface. 
This paper is organized as follows. The geometry of the problem is described in section 2 along with a commonly accepted description of the statistical properties of the sidewall roughness. In section 3 the formulation of the $n_{\mathrm{w}}$ model for radiative losses is presented and compared to the Lacey-Payne model (whose complete formulation is reported in appendix A) for $2 \mathrm{D}$ waveguides. A comparison with experimental results on channel SOI waveguides is presented, showing the validity of the $n_{\mathrm{w}}$ model for 3D structures. In section 4 the $n_{\mathrm{w}}$ model is applied to the investigation of backscattering, proving the equivalence with the Ladouceur-Poladian model (for 2D structures) and the agreement with experimental results on different waveguide technologies and geometries. In section 5 a discussion on the sensitivity of $n_{\mathrm{eff}}$ with respect to the waveguide width and the waveguide parameters is presented. Appendix B reports the analytical derivation of the scaling factors required by the $n_{\mathrm{w}}$ model for both radiative losses and backscattering.

\section{Extrinsic loss in optical waveguides}

The attenuation experienced by the mode propagating in an optical waveguide is related to different contributions. Assuming a weak attenuation, the power insertion loss of a waveguide of length $L_{\mathrm{w}}$ can be written as

$$
\mathrm{IL}=\mathrm{e}^{\alpha L_{\mathrm{w}}}=\mathrm{e}^{\left(\alpha_{\mathrm{a}}+\alpha_{\mathrm{r}}+\alpha_{\mathrm{b}}\right) L_{\mathrm{w}}}
$$

where $\alpha_{\mathrm{a}}$ takes into account the pure material absorption while $\alpha_{\mathrm{r}}$ and $\alpha_{\mathrm{b}}$ describe the fraction of the light coupled to radiative modes and counter-propagating modes (backscattering), respectively. As $\alpha_{\mathrm{r}}$ and $\alpha_{\mathrm{b}}$ are associated with sidewall roughness, they represent the extrinsic loss contribution. Since $\alpha_{\mathrm{b}}$ is generally small compared to $\alpha_{\mathrm{a}}$ and $\alpha_{\mathrm{r}}$, the previous equation can be approximated as

$$
\mathrm{IL} \simeq\left(1-\alpha_{\mathrm{b}} L_{\mathrm{w}}\right) \mathrm{e}^{\left(\alpha_{\mathrm{a}}+\alpha_{\mathrm{r}}\right) L_{\mathrm{w}}}
$$

where $\alpha_{\mathrm{b}}$ corresponds to the parameter $r_{\mathrm{b}}$ commonly used to describe the backscattered power per unit length.

For the 2D case, the geometry of the problem exploited for the analysis of the extrinsic losses is represented in figure 1 as a symmetric slab waveguide of width $w$ and core and cladding refractive indices $n_{1}$ and $n_{2}$, respectively. The random waveguide sidewall perturbation is described by the standard deviation of the roughness $\sigma$ and a correlation length $L_{\mathrm{c}}$. The statistical behaviour of the roughness is defined through a zero-mean random function $f(z)$ which measures the deviation of the waveguide width from the designed value $w$ at a position $z$ along the propagation direction.

A commonly accepted model to describe the statistical properties of $f(z)$ for lithographically defined waveguides relies on an exponential autocorrelation function [5, 7-10]

$$
R\left(u_{z}\right)=\left\langle f(z) f\left(z-u_{z}\right)\right\rangle=\sigma^{2} \exp \left(\frac{-u_{z}}{L_{\mathrm{c}}}\right)
$$

where $u_{z}$ is the displacement along the direction $z .\langle\cdots\rangle$ represents the ensemble average. This model is implicitly assumed for all the results presented in this paper.

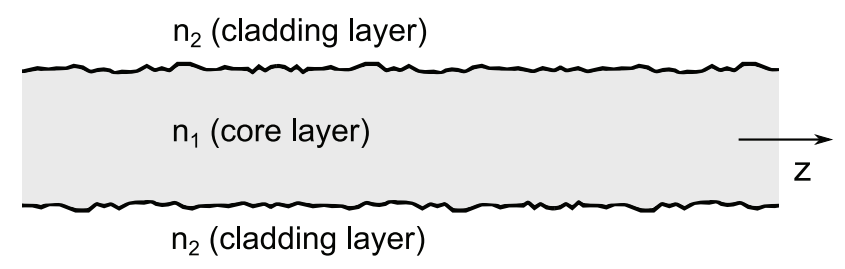

Figure 1. Schematic of a 2D symmetrical slab waveguide with sidewall roughness. The light propagates in the $z$ direction.

\section{Radiation loss model}

The Lacey-Payne model is one of the most generally accepted methods in the literature for the estimation of the radiative losses $\alpha_{\mathrm{r}}$. The computation of the radiated field is based on the method of equivalent currents and assumes the waveguide to behave as a radiating antenna, with the sidewall roughness acting as an equivalent current source [2]. The exponential radiation loss coefficient $\alpha_{\mathrm{r}}$ is then derived for a symmetric single-mode slab waveguide

$$
\alpha_{\mathrm{r}}=\frac{\sigma^{2}}{\sqrt{2} k_{0}(w / 2)^{4} n_{1}} g f
$$

where $k_{0}=2 \pi / \lambda$ is the wavenumber. The $g$ and $f$ functions (whose complete definitions are reported in appendix A) describe how different waveguide parameters contribute to radiative losses. For a given frequency and refractive index profile, the function $g$ is determined only by the waveguide geometry; $f$ is related to the correlation length of the roughness and takes into account the interaction between the propagating mode and the sidewall perturbation. Both functions also depend on the mode's effective refractive index $\left(n_{\text {eff }}\right)$ through the propagation constant $\beta=k_{0} n_{\mathrm{eff}}$.

The expression for $\alpha_{\mathrm{r}}$ in equation (4) does not explicitly highlight the fundamental role played by $n_{\mathrm{eff}}$ and $w$ in the radiative losses experienced by the propagating mode as a consequence of sidewall roughness. As demonstrated in appendix B, a reformulation of this expression reveals that the losses predicted by equation (4) as function of the waveguide width match the derivative of the effective refractive index with respect to $w$

$$
\alpha_{\mathrm{r}}=A \frac{\partial n_{\mathrm{eff}}}{\partial w}
$$

The proportionality factor $A$ depends on $w$ only through $n_{\text {eff }}$ and, sufficiently far from the mode's cut-off, can be assumed as independent of the slab width. The rigorous explicit expression for the coefficient $A$ is reported in appendix B. $A$ takes into account the standard deviation of the roughness $\sigma$ and the correlation length $L_{\mathrm{c}}$ and depends directly on the wavelength $\lambda$. Since the effective refractive index is the main parameter describing the confinement of the mode inside the waveguide core, the derivative in equation (5) gives information on the 'sensitivity' of the mode to the width variations produced by the sidewall roughness and consequently on the amount of power coupled out to the radiative modes. For this reason this model is referred to as the $n_{\mathrm{w}}$ model. 


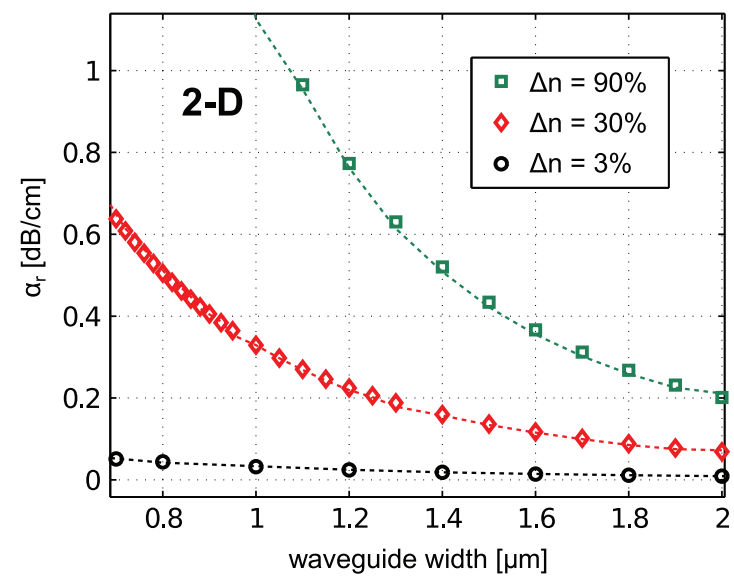

(a)

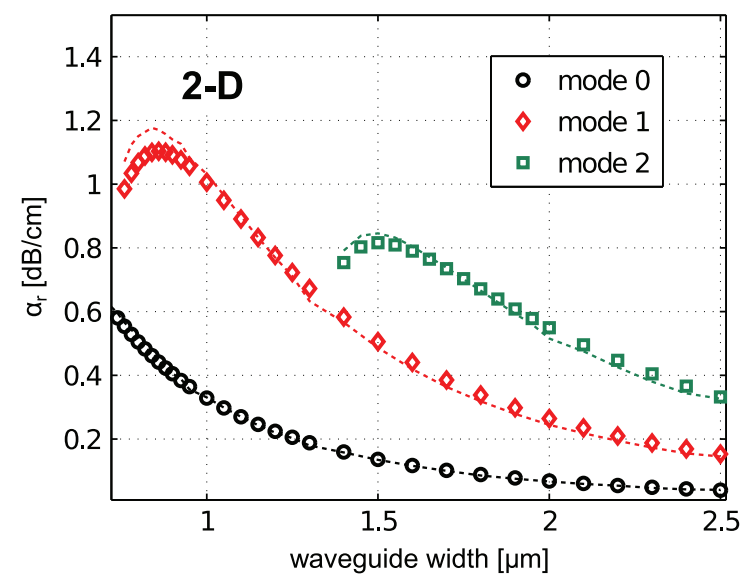

(b)

Figure 2. Comparison between the losses predicted by the Payne-Lacey model (symbols) and the $n_{\mathrm{w}}$ model (dashed lines) for (a) slabs with different index contrast and fixed roughness parameters $\left(\sigma=2 \mathrm{~nm}, L_{\mathrm{c}}=50 \mathrm{~nm}\right)$ and (b) different modes of the slab with $\Delta n=30 \%\left(\sigma=2 \mathrm{~nm}, L_{\mathrm{c}}=50 \mathrm{~nm}\right)$.

Figure 2 shows a comparison between the Payne-Lacey and $n_{\mathrm{w}}$ models for a variety of slab geometries and propagating modes with roughness parameters $\sigma=2 \mathrm{~nm}$ and $L_{\mathrm{c}}=50 \mathrm{~nm}$. For the $n_{\mathrm{w}}$ model, $A$ is assumed as constant (independent of $w)$. In figure 2(a) the loss coefficient $\alpha_{\mathrm{r}}$ as a function of the waveguide width $w$ predicted by both equation (4) (symbols) and equation (5) (dashed lines) is shown for the TE fundamental mode of three different slabs. The waveguides differ in the index contrast between core and cladding, $\Delta n=\left(n_{1}-n_{2}\right) / n_{2}$, which changes from $\Delta n=3 \%$ (e.g. glass waveguides, black circles) to $\Delta n=30 \%$ (e.g. SiN waveguides, red diamonds) and $\Delta n=90 \%$ (high-index-contrast technology, green squares). In all three cases the results are well superposed.

In figure 2(b) the loss coefficient $\alpha_{\mathrm{r}}$ has been calculated only for the waveguide with $\Delta n=30 \%$ with the same roughness parameters as the previous example. In this case a particularly wide slab has been considered in order to evaluate the models' predictions for different propagating modes. For the fundamental (black circles), first (red diamonds) and second (green squares) higher order modes the agreement is excellent, apart from a slight difference near the modes' cut-off. In this point the assumption of a constant value for $A$ is less accurate

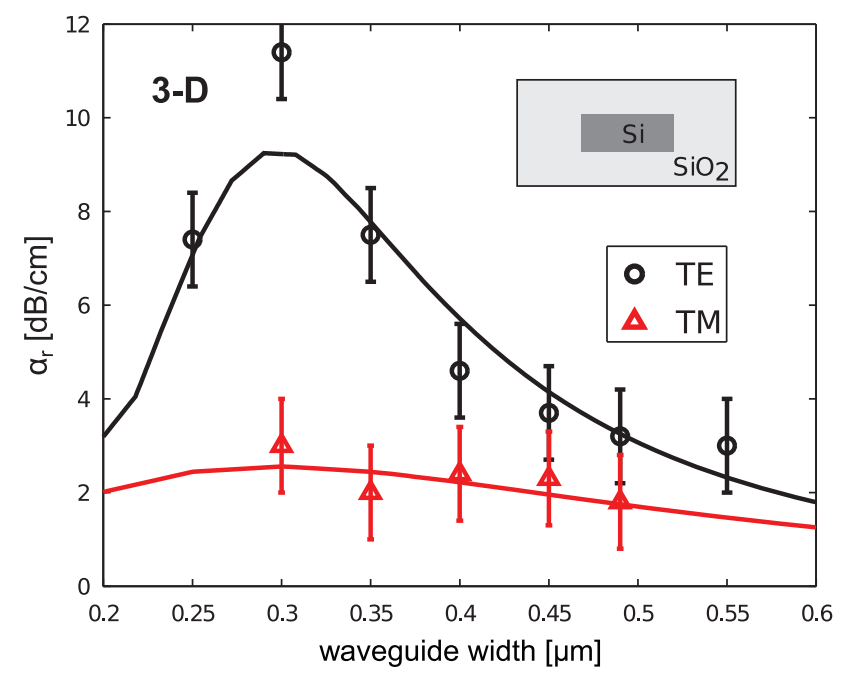

Figure 3. The measured propagation loss (symbols) and the $n_{\mathrm{W}}$ model (solid lines) for channel SOI waveguides (inset) with different widths for TE (black circles) and TM (red triangles) polarized light. The values of the fitting parameters are $A_{\mathrm{TE}}=223.0$ and $A_{\mathrm{TM}}=284.4$.

since $n_{\text {eff }}$ may depend significantly on $w$. For this reason the match between the Lacey-Payne model and the $n_{\mathrm{w}}$ model with constant $A$ results to be less rigorous. The agreement between the two models holds also for TM polarization (not shown for brevity).

Even though the Payne-Lacey model was originally developed only for 2D slab waveguides, it has been applied also to $3 \mathrm{D}$ structures through the effective index method $[8,9,11]$. Good agreement between the numerical model and the experimental results was observed also in these cases. The proposed $n_{\mathrm{w}}$ model holds for 3D waveguides as well. By using the Marcatili separation of variables approximation for a rectangular waveguide of width $w$ and thickness $h$ [12], equation (5) can be generalized to 3D structures as

$$
\alpha_{\mathrm{r}}=A \frac{\partial n_{\mathrm{eff}}}{\partial \mathbf{n}} \simeq A^{\prime} \frac{\partial n_{\mathrm{eff}}}{\partial w}+A^{\prime \prime} \frac{\partial n_{\mathrm{eff}}}{\partial h}
$$

where $\mathbf{n}$ is the normal versor to the waveguide boundaries and $A^{\prime}$ and $A^{\prime \prime}$ take into account the different roughnesses of the vertical and horizontal surfaces of the waveguide. However, the contribution to the radiative loss generated by the roughness of the top/bottom surfaces is typically negligible compared to the sidewall roughness, and the 3D model (6) simply reduces to equation $(5)\left(A^{\prime \prime}=0\right)$. This result is well in accordance with those reported by Yap et al in [11]. As for the slab waveguide, for 3D laterally confined waveguides the parameters $A^{\prime}$ and $A^{\prime \prime}$ can also be considered independent of $w$ and $h$. Equation (5) hence provides a simple and accurate fitting rule for both fundamental and higher order modes of $2 \mathrm{D}$ and 3D waveguides.

Figure 3 shows the measurements of the propagation losses for SOI channel waveguides with $h=220 \mathrm{~nm}$ and increasing $w$ [13] for both the TE (black circles) and TM (red triangles) fundamental modes. The effective index of the modes as a function of the waveguide width has been calculated with a commercial mode solver (FMM method). Neglecting the roughness on the top/bottom surfaces $\left(A^{\prime \prime}=0\right)$, 


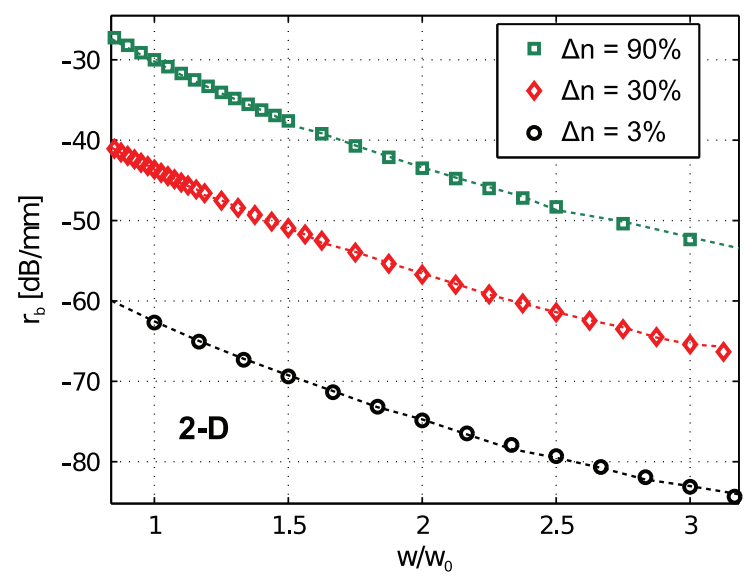

Figure 4. Comparison between the backscattered power predicted by the Ladouceur-Poladian model [6] (symbols) and the $n_{\mathrm{W}}$ model (dashed lines). The backscattering is shown as a function of the waveguide width normalized to the width of the single-mode operation limit $\left(w_{0}\right)$. The same three slab waveguides as in figure 2 (increasing index contrast $\Delta n$ ) with the same roughness parameters $\left(\sigma=2 \mathrm{~nm}, L_{\mathrm{c}}=50 \mathrm{~nm}\right)$ have been used as examples. The values of $w_{0}$ in the three cases are $1.2 \mu \mathrm{m}(\Delta n=3 \%), 0.8 \mu \mathrm{m}(\Delta n=30 \%)$ and $0.4 \mu \mathrm{m}(\Delta n=90 \%)$.

the experimental results have been fitted with equation (5), where the only free fitting parameter is represented by the factor $A$, assumed as width-independent. A good match can be observed between the experimental data and the $n_{\mathrm{w}}$ model for both modes. This is true also in the region around $w=$ $0.3 \mu \mathrm{m}$ where the model predicts a strong enhancement of the propagation losses for the TE polarized mode.

\section{Backscattering model}

The second loss effect originated by the interaction of the guided field with the sidewall imperfections is backscattering. Similarly to radiative losses, a model to evaluate the backscattered signal generated by the sidewall roughness was proposed by Ladouceur and Poladian [6] for a 2D waveguide. The geometry of the problem is the same as figure 1. As in the radiation loss case, a roughness profile with an exponential correlation function given by equation (3) is considered. The backscattering problem is defined in terms of a system of two coupled equations describing the power exchange between the propagating and counter-propagating modes. Assuming a slab of length $L \gg L_{\mathrm{c}}$ and a small perturbation of the sidewalls (with uncorrelated perturbations on the two sidewalls), the distributed power backscatter coefficient can be expressed as [6]

$$
r_{\mathrm{b}}=\left[\frac{U^{2} W}{2(w / 2)^{3} \beta(1+W)}\right]^{2} \frac{\sigma^{2} L_{\mathrm{c}}}{\pi} \frac{1}{1+4 \beta^{2} L_{\mathrm{c}}^{2}} .
$$

The coefficients $U$ and $W$ are defined in appendix A. Assuming weak backscattering, the total power reflected by the waveguide is $r_{\mathrm{b}} L$.

Interestingly, the $n_{\mathrm{w}}$ model proposed for the estimation of the radiation losses can be applied also to the investigation of the backscattering phenomenon [13,14]. As described in appendix $\mathrm{B}$, the model in this case relies on $\partial n_{\mathrm{eff}} / \partial w$ squared instead of the linear dependence of equation (5) for radiative

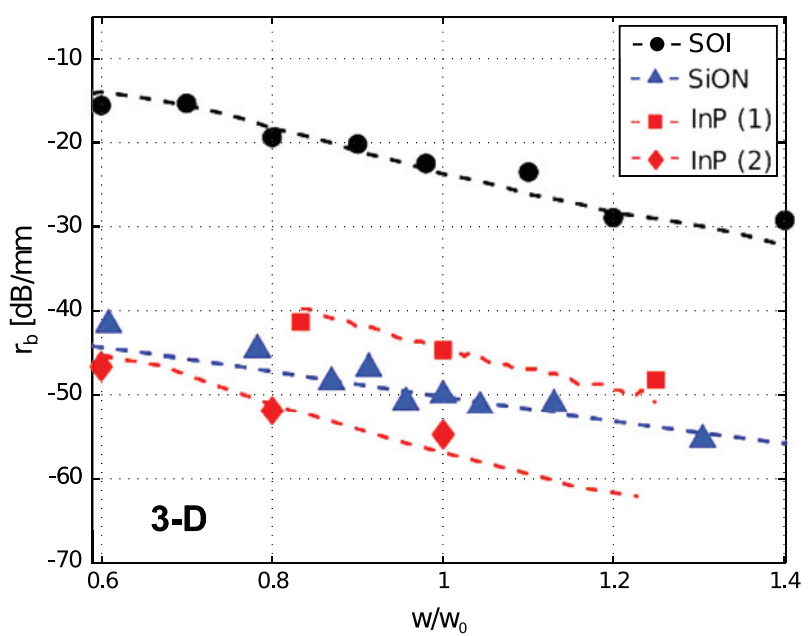

Figure 5. The measured (symbols) backscattering as a function of the normalized waveguide width for several technologies. The experimental data refer to the TE mode and are numerically fitted (dashed lines) with the $n_{\mathrm{w}}$ model. The considered technologies are SOI (black circles, $B=1.73 \times 10^{3}(\mathrm{~nm})$ ) [13], SiON (blue triangles, $\left.B=8.0 \times 10^{4}(\mathrm{~nm})\right)$ [15], rib InGaAsP waveguide (red diamonds, $B=1.49 \times 10^{4}(\mathrm{~nm})$ ) [16], ridge InP waveguide (red squares, $\left.B=1.24 \times 10^{4}(\mathrm{~nm})\right)$ [17].

losses,

$$
r_{\mathrm{b}}=B\left(\frac{\partial n_{\mathrm{eff}}}{\partial w}\right)^{2} .
$$

The expression for the proportionality factor $B$ is given in appendix $\mathrm{B}$. As for the factor $A, B$ also depends on the waveguide width only through $n_{\text {eff }}$ and can be considered independent of $w$ sufficiently far from the modes' cut-off.

Figure 4 shows a comparison between the power backscatter coefficient $r_{\mathrm{b}}$ predicted by the Ladouceur-Poladian model (equation (7), symbols) and the $n_{\mathrm{w}}$ model of equation (8) (dashed lines). The three cases refer to the same slabs as in figure 2(a) for the TE fundamental mode. The waveguide width is normalized to the width limit for single-mode waveguides $\left(w_{0}\right)$ for convenience of presentation. The results are well superposed, suggesting that the two models are essentially equivalent. As expected, higher index contrast increases the sensitivity of the mode to the sidewall imperfection (as in the case of the radiative losses) and consequently the backscattering coefficient. On increasing $\Delta n$ from 3\% to $90 \%$ the backscattered light increases by about $30 \mathrm{~dB}$ almost independently of $w$, becoming a serious problem for high-index-contrast waveguides.

As for the radiation losses, the $n_{\mathrm{w}}$ model can also be applied to 3D laterally confined waveguides through the derivative $\partial n_{\text {eff }} / \partial \mathbf{n}$. Neglecting the contribution of the roughness on the top/bottom surfaces of the waveguide, equation (8) is valid in the 3D case as well, as suggested also in [13], using $B$ as the fitting parameter for the backscattering data. Figure 5 shows the measured backscattered power (symbols) as a function of the waveguide width (TE input mode) for four very different types of waveguides: the silicon wire in SOI technology used in figure 3 [13], a $2 \mu \mathrm{m}$-thick channel SiON waveguide [15], a rib waveguide with an InGaAsP-based core (thickness $1 \mu \mathrm{m}$ ) on an InP substrate and no cladding [16] and a 
deeply etched InP-based ridge waveguide with a $360 \mathrm{~nm}$-thick multi-quantum well core [17]. The experimental data are fitted with the $n_{\mathrm{w}}$ model (dashed lines), which holds correctly for all the different technologies.

\section{Discussion}

Some considerations are worthwhile with regard to the relation between the $n_{\mathrm{w}}$ model and the waveguide parameters. Both the Payne-Lacey model and the Ladouceur-Poladian model, applied to either $2 \mathrm{D}$ slab or $3 \mathrm{D}$ waveguides through the effective index method, can be used to predict the loss coefficient $\alpha_{\mathrm{r}}$ and the backscattering $r_{\mathrm{b}}$ as function of the waveguide width once the information about the waveguide geometry and sidewall roughness has been provided. The examples of the previous sections show how, in both 2D and $3 \mathrm{D}$ cases, these results are correctly matched by those obtained by applying the $n_{\mathrm{w}}$ model, which basically represents the derivative of the mode's effective index with respect to the waveguide width, apart from a constant scaling factor.

Considering a symmetric slab with core index $n_{1}$, cladding index $n_{2}$ and width $w$ (figure 1), the normalized frequency can be defined as

$$
V=\frac{\omega}{c} w \sqrt{n_{1}^{2}-n_{2}^{2}}
$$

where $\omega$ is the angular frequency and $c$ is the speed of light. Taking the derivative of $n_{\text {eff }}$ with respect to $\omega$ and $w$ and considering equation (9) leads to

$$
w \frac{\partial n_{\mathrm{eff}}}{\partial w}=\omega \frac{\partial n_{\mathrm{eff}}}{\partial \omega}=-\lambda \frac{\partial n_{\mathrm{eff}}}{\partial \lambda} .
$$

Combining equation (10) with the definition of the group effective index $n_{\mathrm{g}}=n_{\text {eff }}+\omega\left(\partial n_{\text {eff }} / \partial \omega\right)$ and assuming nondispersive materials $\left(\partial n_{1 / 2} / \partial \omega=0\right)$ the following result is found:

$$
\frac{\partial n_{\mathrm{eff}}}{\partial w}=\frac{n_{\mathrm{g}}-n_{\mathrm{eff}}}{w} .
$$

Equation (11) relates the $n_{\mathrm{w}}$ model to the difference $\left(n_{\mathrm{g}}-n_{\mathrm{eff}}\right)$. The dependence of the waveguide losses and backscattering on the difference $\left(n_{\mathrm{g}}-n_{\mathrm{eff}}\right)$ is rigorous and is related to the fact that a change in the waveguide width modifies both the group index and the mode's field distribution and hence the interaction with the sidewall roughness.

In the case of 3D waveguides, equation (11) still holds provided that the derivative $\partial n_{\text {eff }} / \partial \mathbf{n}$ is considered. In more detail, the difference between $n_{\mathrm{g}}$ and $n_{\mathrm{eff}}$ is related to the relative strength of the longitudinal component of the field with respect to the transverse component [18]. This can be shown by introducing the time-averaged power $P$ transported by the waveguide

$$
P=\int_{-\infty}^{+\infty} \mathrm{d} x \mathrm{~d} y S_{z}=\int_{-\infty}^{+\infty} \mathrm{d} x \mathrm{~d} y\left[\mathbf{E} \times \mathbf{H}^{*}+\mathbf{E}^{*} \times \mathbf{H}\right]_{z} .
$$

Here, $S_{z}$ is the component of the Poynting vector in the propagation direction which depends only on the transverse component of the field. The integration is made on the whole cross-section of the waveguide. With a variational approach (and assuming the absence of material dispersion) the following relation is demonstrated [18]:

$$
\left(n_{\mathrm{g}}-n_{\mathrm{eff}}\right)=\frac{2 c}{P} \int_{-\infty}^{+\infty} \mathrm{d} x \mathrm{~d} y\left[\mathbf{E}_{z} \cdot \mathbf{E}_{z}^{*}+\mathbf{H}_{z} \cdot \mathbf{H}_{z}^{*}\right]
$$

where the subscript $z$ refers to the field component in the propagation direction and $c$ is the speed of light. Equation (13) states that the difference between $n_{\mathrm{g}}$ and $n_{\text {eff }}$ goes to zero when the longitudinal component of the propagating mode vanishes, for example with small refractive index contrast $\Delta n$ or when the waveguide is strongly multimode [18]. In this regime the sensitivity of the field to the sidewall roughness vanishes as well (since $\partial n_{\text {eff }} / \partial w \rightarrow 0$ ) and backscattering and radiative losses become negligible.

\section{Conclusions}

In conclusion, we have demonstrated that in optical waveguides affected by sidewall roughness both the radiative losses and the backscattering depend on the sensitivity of the waveguide mode's effective index to the width of the waveguide, that is on the derivative $\partial n_{\text {eff }} / \partial w$ ( $n_{\mathrm{w}}$ model). The $n_{\mathrm{w}}$ model applies to a very good approximation to $3 \mathrm{D}$ structures with arbitrary waveguide geometries, irrespective of the considered photonic technology, as proven by the agreement with experimental results.

Compared to existing and established approaches [2, 6], the $n_{\mathrm{w}}$ model enables an easier understanding of the interaction between the sidewall roughness and the optical field, thereby providing straightforward design rules, directly related to the geometric and optical parameters of the waveguide, for minimization of the extrinsic loss. In particular, both $\alpha_{\mathrm{r}}$ and $r_{\mathrm{b}}$ reduce when $\partial n_{\text {eff }} / \partial w \rightarrow 0$, that is when the group index $n_{\mathrm{g}}$ approaches $n_{\text {eff }}$. This condition is typically difficult to reach in high-index-contrast technologies, thus explaining the loss [3-5] and backscattering [13] issues of silicon photonic waveguides compared to low-index-contrast waveguides.

Finally, the key role pointed out here for the group index and the mode shape (through the $n_{\text {eff }}$ parameter) in the scattering processes of classical waveguides finds a strong analogy in the extrinsic loss problem of disordered photonic crystal waveguides [19]. In both kinds of structures the radiation loss and backscattering scale (approximately) with $n_{\mathrm{g}}$ and $n_{\mathrm{g}}^{2}[19,20]$, respectively, these relations setting tight constraints on the optimization of the waveguide design.

\section{Acknowledgment}

This work was partially supported by the European Community's Seventh Framework Programme FP7/2007-2013 under Grant ICT 257210 (PARADIGM).

\section{Appendix A. The Lacey-Payne model}

We recall in this appendix the complete expressions for the $g$ and $f$ functions of the Lacey-Payne model as reported in [2] for a symmetric slab waveguide. The function $g$ is defined as

$$
g(V)=\frac{U^{2} V^{2}}{1+W}
$$


with the parameters defined as

$$
U=\frac{w}{2} \sqrt{n_{1}^{2} k_{0}^{2}-\beta^{2}}, \quad W=\frac{w}{2} \sqrt{\beta^{2}-n_{2}^{2} k_{0}^{2}}
$$

and $V$ defined by equation (9). $n_{1}$ and $n_{2}$ are the core and cladding refractive indices, respectively, of a slab waveguide of width $w . k_{0}$ is the wavenumber and $\beta$ is the propagation constant. The definition of the function $f$ is

$$
\begin{aligned}
f(x, \gamma) & =x \tilde{f}(x, \gamma) \\
& =\frac{x \sqrt{\left[\left(1+x^{2}\right)^{2}+2 x^{2} \gamma^{2}\right]^{1 / 2}+1-x^{2}}}{\sqrt{\left(1+x^{2}\right)^{2}+2 x^{2} \gamma^{2}}}
\end{aligned}
$$

where

$x=W \frac{L_{\mathrm{c}}}{w / 2}, \quad \gamma=\frac{n_{2} V}{n_{1} W \sqrt{\Delta}}, \quad \Delta=\frac{n_{1}^{2}-n_{2}^{2}}{2 n_{1}^{2}}$

with $L_{\mathrm{c}}$ the correlation length of the sidewall roughness.

\section{Appendix B. Demonstration of equations (5) and (8)}

From the definitions in appendix $\mathrm{A}$ it is clear that both of the parameters $x$ and $\gamma$ of the Lacey-Payne model do not depend directly on the slab width $w$ but only through the effective refractive index $n_{\text {eff }}=\beta / k_{0}$. The same is true also for the function $f(x, \gamma)$. In particular, a numerical analysis shows that for most practical cases $f(x, \gamma)$ (and hence $\tilde{f}(x, \gamma))$ is a linear function of the effective refractive index. Since far from the cut-off of the mode $n_{\text {eff }}$ is almost constant with the waveguide width (in particular for low-index-contrast waveguides), both $\tilde{f}(x, \gamma)$ and $f(x, \gamma)$ exhibit a very slight dependence on $w$.

Rearranging the terms of equation (4) and exploiting appendix A, a direct dependence on the slab width in the Lacey-Payne model can be highlighted,

$$
\alpha_{\mathrm{r}}=C \frac{1}{1+(w / 2) k_{0} \sqrt{n_{\mathrm{eff}}^{2}-n_{2}^{2}}} .
$$

The factor $C$ is defined as

$$
C=\frac{\sigma^{2} k_{0}^{3}\left(n_{1}^{2}-n_{2}^{2}\right)}{\sqrt{2} n_{1}} f\left(n_{1}^{2}-n_{\mathrm{eff}}^{2}\right)
$$

and does not depend explicitly on $w$.

The derivative of the effective index with respect to the waveguide width exploited in the $n_{\mathrm{w}}$ model can be calculated for a symmetric slab through equation (16) of [21]. With simple manipulation

$$
\frac{\partial n_{\mathrm{eff}}}{\partial w}=\frac{n_{1}^{2}-n_{\mathrm{eff}}^{2}}{\tilde{w} n_{\mathrm{eff}}} .
$$

The effective width of the waveguide $\tilde{w}$ is defined for a symmetric slab as

$$
\tilde{w}=w+\frac{2}{k_{0} \sqrt{n_{\mathrm{eff}}^{2}-n_{2}^{2}}} .
$$

Substituting equation (B.4) into equation (B.3) the derivative results to be

$$
\frac{\partial n_{\mathrm{eff}}}{\partial w}=D \frac{1}{1+(w / 2) k_{0} \sqrt{n_{\mathrm{eff}}^{2}-n_{2}^{2}}}
$$

with

$$
D=\frac{\left(n_{1}^{2}-n_{\text {eff }}^{2}\right) k_{0} \sqrt{n_{\text {eff }}^{2}-n_{2}^{2}}}{2 n_{\text {eff }}} .
$$

As for the factor $C, D$ also depends on $w$ only through the effective refractive index $n_{\mathrm{eff}}$.

The propagation losses predicted by the Lacey-Payne model are then equivalent to the derivative of the effective refractive index with respect to the slab width $\left(n_{\mathrm{w}}\right.$ model)

$$
\alpha_{\mathrm{r}}=A \frac{\partial n_{\mathrm{eff}}}{\partial w}
$$

through the proportionality factor A which can finally be calculated as

$$
A=\frac{C}{D}=\frac{\sigma^{2} L_{\mathrm{c}} k_{0}^{3}\left(n_{1}^{2}-n_{2}^{2}\right) \sqrt{2}}{n_{1}} n_{\mathrm{eff}} \tilde{f} .
$$

As already stated, far from the mode's cut-off the term $n_{\text {eff }} \tilde{f}$ can be considered almost independent of the waveguide width, as demonstrated by the results in figure 2 where $A$ has been assumed as constant. A deviation of the $n_{\mathrm{w}}$ model from the Lacey-Payne results can be observed near the cut-offs of the modes (e.g. the first and second higher order modes in figure 2(b)), where even a small increase of the waveguide width provokes a non-negligible variation of $n_{\text {eff }}$ (and hence $A$ ).

The $n_{\mathrm{w}}$ model for backscattering can be demonstrated with similar arguments exploiting equations (B.5) and (B.6) to compute the expression of $\left(\partial n_{\mathrm{eff}} / \partial w\right)^{2}$. Comparing this result with equation (7), the coefficient $B$ of equation (8) is defined as

$$
B=\frac{\sigma^{2} L_{\mathrm{c}} k_{0}^{2}}{\pi\left(1+4 k_{0}^{2} L_{\mathrm{c}}^{2} n_{\mathrm{eff}}\right)} .
$$

As for the proportionality factor $A, B$ also depends on the waveguide width only through the effective refractive index $n_{\text {eff. }}$.

\section{References}

[1] Marcuse D 1969 Radiation losses of dielectric waveguides in terms of the power spectrum of the wall distortion function Bell Syst. Tech. J. 48 3233-42

[2] Payne F P and Lacey J P R 1994 A theoretical analysis of scattering loss from planar optical waveguides $O p t$. Quantum Electron. 26 977-86

[3] Lee K K, Lim D R, Kimerling L C, Shin J and Cerrina F 2001 Fabrication of ultralow-loss $\mathrm{Si} / \mathrm{SiO}_{2}$ waveguides by roughness reduction Opt. Lett. 26 1888-90

[4] Vlasov Y and McNab S 2004 Losses in single-mode silicon-on-insulator strip waveguides and bends $O p t$. Express 12 1622-31

[5] Poulton C G, Koos C, Fujii M, Pfrang A, Schimmel T, Leuthold J and Freude W 2006 Radiation modes and roughness loss in high index-contrast waveguides IEEE $J$. Sel. Top. Quantum Electron. 12 1306-21 
[6] Ladouceur F and Poladian L 1996 Surface roughness and backscattering Opt. Lett. 21 1833-5

[7] Ladouceur F, Love J D and Senden T J 1994 Effect of side wall roughness in buried channel waveguides IEE Proc.Optoelectron. $141242-8$

[8] Lee K K, Lim D R, Luan H-C, Agarwal A, Foresi J and Kimerling L C 2000 Effect of size and roughness on light transmission in a $\mathrm{Si} / \mathrm{SiO}_{2}$ waveguide: experiments and model Appl. Phys. Lett. 77 1617-9

[9] Grillot F, Vivien L, Laval S, Pascal D and Cassan E 2004 Size influence on the propagation loss induced by sidewall roughness in ultrasmall SOI waveguides IEEE Photon Technol. Lett. 16 1661-3

[10] Barwicz T and Haus H 2005 Three-dimensional analysis of scattering losses due to sidewall roughness in microphotonic waveguides J. Lightwave Technol. 23 2719-32

[11] Yap K P, Delage A, Lapointe J, Lamontagne B, Schmid J H, Waldron P, Syrett B A and Janz S 2009 Correlation of scattering loss, sidewall roughness and waveguide width in silicon-on-insulator (SOI) ridge waveguides J. Lightwave Technol. 27 3999-4008

[12] Marcatili E A J 1969 Dielectric rectangular waveguide and directional coupler for integrated optics Bell Syst. Tech. J. 48 2071-102

[13] Morichetti F, Canciamilla A, Ferrari C, Torregiani M, Melloni A and Martinelli M 2010 Roughness induced backscattering in optical silicon waveguides Phys. Rev. Lett. 104033902
[14] Verly P, Tremblay R and Lit J W Y 1980 Application of the effective-index method to the study of distributed feedback in corrugated waveguides: TM polarization J. Opt. Soc. Am. $701218-21$

[15] Morichetti F, Melloni A, Breda A, Canciamilla A, Ferrari C and Martinelli M 2007 A reconfigurable architecture for continuously variable optical slow-wave delay lines Opt. Express 15 17273-82

[16] Melati D, Morichetti F, Canciamilla A, Roncelli D, Soares F M, Bakker A and Melloni A 2012 Validation of the building-block-based approach for the design of photonic integrated circuits J. Lightwave Technol. $303610-6$

[17] Kleijn E, Williams P J, Whitbread N D, Wale M J, Smit M K and Leijtens X J M 2012 Sidelobes in the response of arrayed waveguide gratings caused by polarization rotation Opt. Express 20 22660-8

[18] Kogelnik H and Weber H P 1974 Rays, stored energy, and power flow in dielectric waveguides J. Opt. Soc. Am. 64 174-85

[19] Hughes S, Ramunno L, Young J F and Sipe J E 2005 Extrinsic optical scattering loss in photonic crystal waveguides: role of fabrication disorder and photon group velocity Phys. Rev. Lett. 94033903

[20] O'Faolain L et al 2010 Loss engineered slow light waveguides Opt. Express 18 27627-38

[21] Kogelnik H and Ramaswamy V 1974 Scaling rules for thin-film optical waveguides Appl. Opt. 13 1857-62 